\title{
Esthetics in Hemimaxillectomy : A Case Report
}

\author{
Dr. Lalit A Manek ${ }^{1}$,Dr. Asha Rathod ${ }^{2}$,Dr.Gaurang Mistry ${ }^{3}$, \\ Dr.Keyuresh Kulkarni ${ }^{4}$
}

\begin{abstract}
The goal of prosthodontics is rehabilitation of missing oral and extraoral structures along with restoration of the normal functions of mastication, speech, swallowing, appearance.Hemimaxillectomy patients present with abrupt alterations in the physiologic processes of the maxillae, along with increased anxiety levels due to sudden impairment in aesthetics, mastication, speech. Such patients should be rehabilitated as early as possible. This case report illustrates the rehabilitation of a patient, who had undergone maxillectomy on the left side, due to maxillary neurilemmoma. The transitional prosthesis given initially followed by a definitive prosthesis using a cast partial denture.
\end{abstract}

Keywords: Hemi maxillectomy, Obturator, Maxillary Neurilemmoma, Aramany Class II defect.

\section{Introduction}

Maxillectomy is a term used to describe the partial or total removal of the maxilla, often performed in patients suffering from benign or malignant neoplasms. The resulting defect may include any portion of the hard and/or soft palate, the alveolar ridges and the floor of the nasal cavity. ${ }^{1}$ Patients with acquired maxillary defects differ from those with congenital defects because of the abrupt alteration in physiologic processes associated with surgical resection of the maxilla.This effect diminishes the patient's quality of life and self-esteem. Prosthodontic rehabilitation of such defect by obturator prosthesis would restore the patient to a normal level of function. ${ }^{2}$ Hemimaxillectomy patients should be restored to normal function as soon as possible postoperatively. Construction of a transitional prosthesis will enable the patient to eat, speak and swallow normally. These are essential functions for physiologic and psychologic health. The patient can then become a functioning member of the society.

The defect could be acquired, developmental.Almost all acquired palatal defects are precipitated by the resection of neoplasms of the palate and paranasalsinuses. The extent of the resection is dependent on the size, location, and potential behaviour of the tumor. ${ }^{3}$ Prosthodontic therapy for patients with acquired surgical defects of the maxilla can be divided into three phases of treatment with each phase having different objectives. ${ }^{4}$

The temporary obturator is constructed from the postsurgical impression cast which has an artificial palate and artificial ridge and generally has no teeth. The closed bulb extending into the defect area is hollow. The patient is usually seen every two weeks because of the rapid soft tissue changes that occur within the defect during the organization and healing of the wound. The new lining material is placed or changed. It is best to remove the entire old interim lining material because of porosity, leading to bacterial contamination and precipitation of undesirable odors and mucosal irritations. The periodic addition of interim lining material increases the bulk and weight of the obturator and this temporary material may become rough and unhygienic.

A definitive obturator is not indicated until the surgical site is healed and dimensionally stable and the patient is prepared physically and emotionally for the restorative care that maybe necessary. Changes associated with healing and remodelling will continue to occur in the border areas of the defect for at least one year. Dimensional changes are primarily related to the peripheral soft tissues rather than to bony support areas Approximately six months after surgery, consideration maybe given to the construction of a definitive obturatorprosthesis. It is constructed from the postsurgical maxillary cast. This obturator has a metal framework which acts as the palate and supports the teeth and the closed bulb which is hollow. Often the temporary obturator will need to function comfortably for as long as six months. The timing will vary depending on the size of the defect, the progress of healing, the prognosisfor control of the tumour, the effectiveness of the presentobturator, and the presence or absence of teeth. Toreduce and improve adhesion and retention, a hollow obturator bulb is required for cleft palate prostheses and for dentures following maxillectomies. ${ }^{5}$

\section{Case Report}

40 -year- old male reported to the department with a swelling localized on her left cheek presented with pain in the left maxillary sinus region since one month. The biopsy revealed a maxillary neurilemmoma which is supposed to be the rarest of the rare tumour, surgical excision of tumour with preservation of palatal flap was performed. He presented for prosthodontic rehabilitation at 4 months interval. On examination,extraorally patients sub-zygomatic region looked depressed and on intraoral examination the left maxillary segment was missing and the palatal mucosa was firm and resilient.This defect corresponded to the Aramany Class II defect. 
Prosthetic obturation with a definitive obturator with the cast metal framework was planned. Patient being aesthetically more conscious, aninterimheat cure prosthesis was planned.

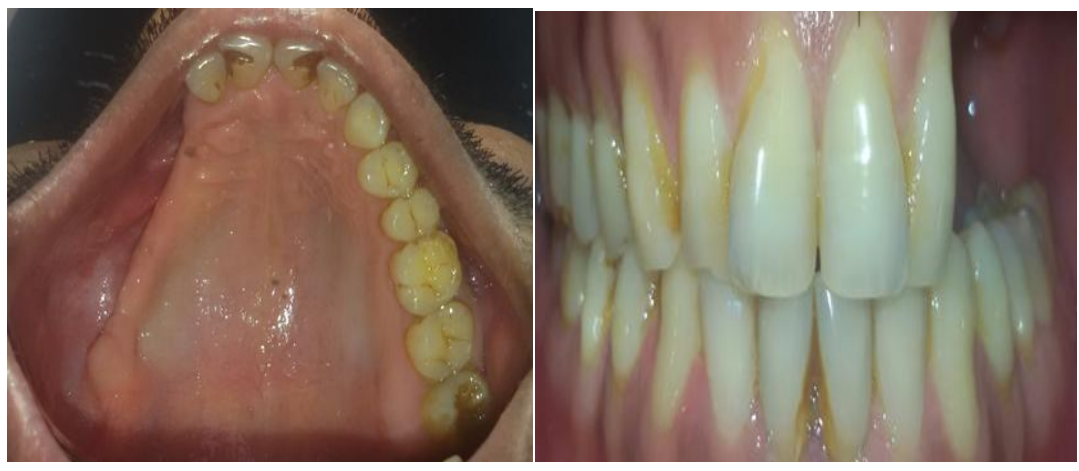

Fig.1 Intraoral view of the maxillary arch
Fig.2 Intraoral view in Maximum Intercuspation

\section{Procedure}

Primary impression was made with irreversible hydrocolloid (Prime chrome).The impression was poured with Type-III dental stone (Kala Stone) to obtain primary cast.A conventional maxillary removable partial denturewas fabricated following the accepted prosthodontic norms; 21 -gauge, round, wrought wire clasps were used for retention on the remaining natural teeth.Patient was using interim prosthesis till the time he was comfortable with it.After the interval of 3 weeks further procedure for cast partial denture was initiated. Preparation of rest seats on 14, 15 and 16, 17 was done to receive embrasure clasp of cast metal framework. Complete palate major connector was designed considering the partial loss of palate.

I- bar was given to the patient initially during trial. Patient was not satisfied with the metal exposure in the anterior region, hence the decision of going for acetal clasp of the tooth shade was taken. On removal of the I-bar,acetal clasp was given on the cervical aspect of the lateral and central.

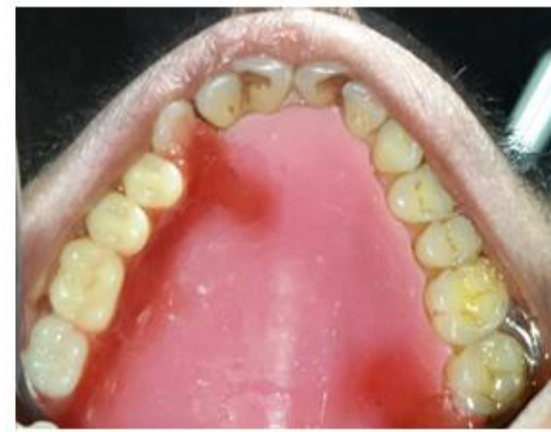

Fig.3 Try in of temporary prosthesis

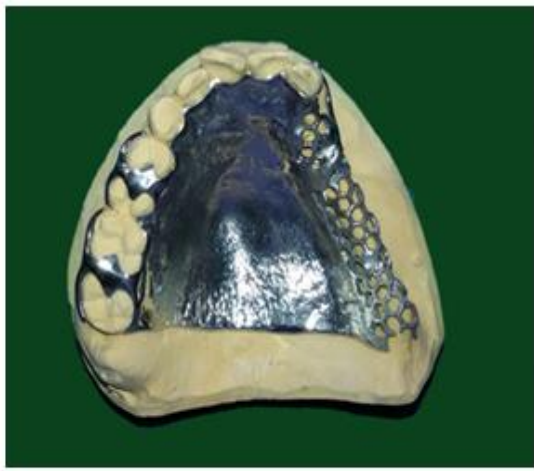

Fig.5 Metal Framework on maxillary

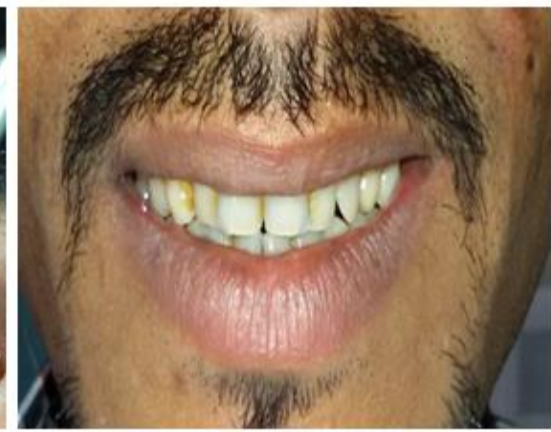

Fig.4 Temporary prosthesis Frontal view

cast

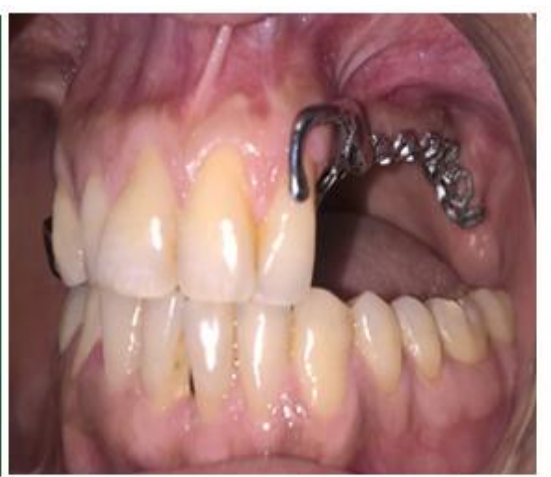

Fig. 6 Framework try in 


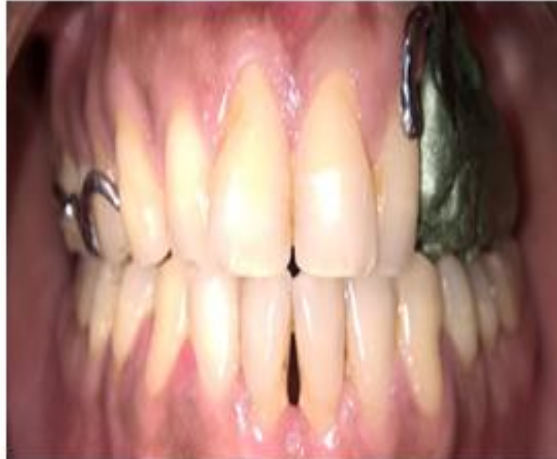

Fig . 7 Bite Registration

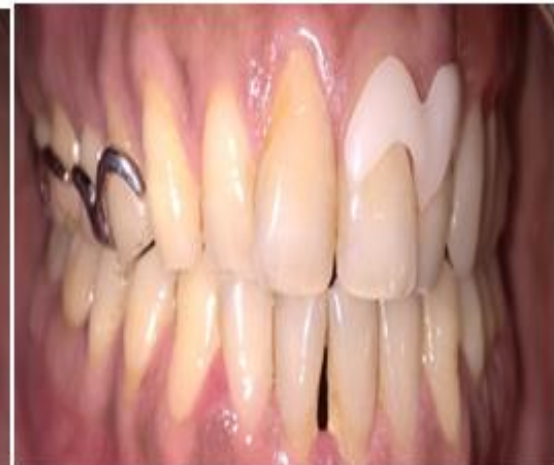

Fig. 8 Acetal clasp

Border moulding was done using low fusing compound and final impression made with medium body impression material (Aquasil Monophase). The master cast was surveyed \& designed for cast partial framework. A cast metal framework was fabricated and checked intra-orally for retention and fit. Alu-Wax occlusal rims were fabricated, Jaw relation recorded and transferred to the articulator. Teeth arrangement done and trial obturator was tried in the patient's mouth. Waxed up obturator was processed using heat cure acrylic resin (Lucitone 199 OR). Insertion was done and was assessed. The patient was satisfied and comfortable with the functioning and aesthetics of the prosthesis.

Highly cross-linked Acrylic resin teeth(Acryrock) were used and a processed acrylic resin denture base was made with the compression moulding technique. Occlusion in cpd.The purpose of this was to reducethe stress on the resected site. The prosthesis was fittedin the mouth. The occlusion was adjusted and the borders checked. The patient was instructed to wear the prosthesis for 1 week. After this period he returned and areas of soreness were relieved. The border of the prosthesis in the region of surgery was then reduced about $1 \mathrm{~mm}$.

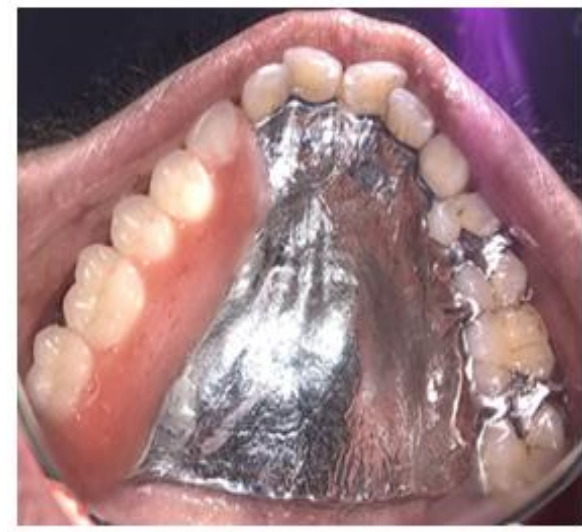

Fig .9 Intraoral view of final prosthesis

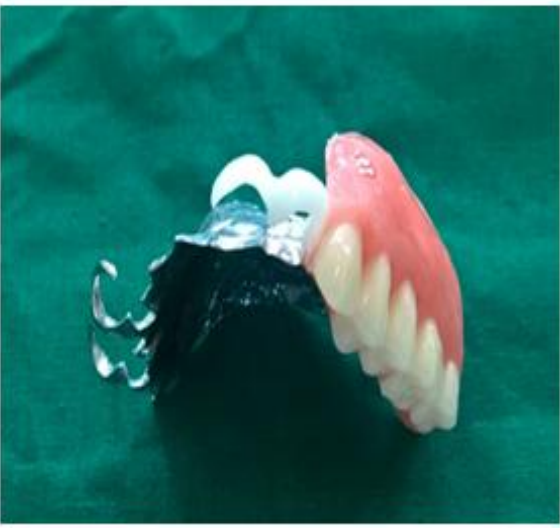

Fig. 10 Acetal clasp on final

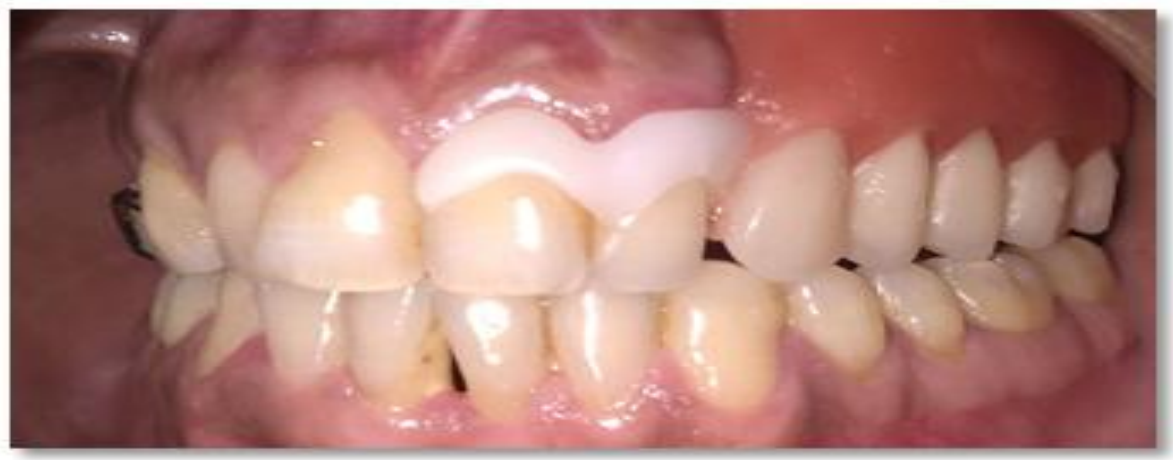

Fig. 11 Final prosthesis 


\section{Discussion}

A definitive obturator is not indicated until the surgical site is healed and dimensionally stable and the patient is prepared physically and emotionally for the restorative care that maybe necessary. The obturator may be displaced superiorly with the stress of mastication and will tend to drop without occlusal contact. The degree of movementwill vary with the number and position of teeth, the size and configuration of the defect, the amount and contour of the remaining palatal area, height of the residual alveolar ridge, the size, contour, and lining mucosa of the defect, and the availability of undercuts. Lack of retention, stability, and support are common problems of treatment for patients who have had a maxillectomy. The aim of oral cancer treatment is not only the preservation of life but also life, after treatment is emphasized. ${ }^{6}$

Oral rehabilitation after hemimaxillectomy presents diverse clinical and technical problems. The usual treatment sequence includes placement of a surgical obturator during the intervention; then 5-10 days later a removable interim obturator is constructed and placed for the duration of the wound healing period; finally, the definitive obturator is constructed and placed about 3-6 months post-surgery, when major changes in tissue conformation are no longer expected. ${ }^{7}$

The primary goal of prosthetic obturation is closure of the maxillectomy defect and separation of the oral cavity from the sino-nasal cavities. A pressure- resistant seal of the obturator bulb against the mucosal lining and skin graft, if placed, restores speech and swallowing functions. A successful prosthetic design for functional restoration of the maxillectomy defect utilizes the remaining palate and dentition to maximize the support, stability, and retention of an obturator bulb.

\section{Refrences}

[1]. Chalian, V.A., Drane, J.B. \& Standish, S.M. Maxillofacial Prosthetics. Multidisciplinary practice, The Williams \& Wilkins Co., Baltimore: 1971; pp. 133-148.

[2]. Ahila SC, Anitha KV, Thulasingam C.Comparison of obturator design for acquired maxillary defect in completely edentulous patients. Indian J Dent Res 2011; 22(1): 161-163.

[3]. Beumer III, Curtis TA, Firtell DN. Maxillofacial rehabilitation. Prosthodontic and surgical considerations. St Louis, Toronto, London:The CV. Mosby Co.; 1979. p. 188-243

[4]. Wiens JP. Acquired maxillofacial defects from motor vehicle accidents: Statistics and prosthodontic considerations. J Prosthet Dent 1990;63:172-81

[5]. Buckner H. Construction of a denture with hollow obturator, lid, and soft acrylic lining. J Prosthet Dent 1974;31:95-9.

[6]. H S Loh, P H Tan. Prosthodontic management of maxillofacial defects after cancer surgery. SING MED J 1989; 30:74-78.

[7]. Waldron JN, O'Sullivan B, Gullane P, Witterick IJ, Liu FF, Payne D, Warde P, Cummings B. Carcinoma of the maxillary antrum: a retrospective analysis of 110 cases. Radiother Oncol 2000; 57: 167-173. 